\title{
Andreas ROTTACH ${ }^{1}$, Micha JUNG \& Thomas MILLER (Kempten)
}

\section{Tandem Teaching - Kooperative Lehre und Einbindung hochschulexterner Lehrpersonen ${ }^{2}$}

\section{Zusammenfassung}

Dieser Werkstattbericht stellt die Konzeption eines für die wissenschaftliche Weiterbildung entwickelten innovativen Lehrformats dar, welches in einem berufsbegleitenden Studiengang an einer Hochschule umgesetzt wird. Die ersten Ergebnisse aus der Erprobungsphase zeigen, dass kooperative Lehrformen verbunden mit der Einbindung von Lehrpersonen aus der Berufspraxis hohen Mehrwert für die Zielgruppe berufserfahrener Studierender haben. Dies gilt allerdings nur unter der Voraussetzung, dass sich die Lehrperson aus der Praxis im selben beruflichen Feld verortet wie die Studierenden.

\section{Schlüsselwörter}

Hochschulexterne Lehrende, kooperative Lehre, berufsbegleitendes Studieren, innovative Lehrformen

${ }^{1}$ E-Mail: andreas.rottach@hs-kempten.de

${ }^{2}$ Dieser Beitrag basiert auf der Forschungstätigkeit im Rahmen des Projekts „Jugendarbeit mit Perspektive (JumP)“ unter der Leitung von Prof. Dr. Peter Nick und Prof. Dr. Patricia Pfeil, gefördert durch das deutsche Bundesministerium für Bildung und Forschung (BMBF) unter dem Förderkennzeichen 16OH22028 im Rahmen des Bund-LänderWettbewerbs „Aufstieg durch Bildung: offene Hochschulen“.

\section{(cc) BY-NC-ND}




\title{
Tandem teaching - Cooperative teaching in higher education with integration of external practitioners
}

\begin{abstract}
In this paper, the authors discuss their experience developing and testing a concept for an innovative teaching approach for continuing education at higher education institutions. A part-time university course that uses this concept is evaluated. Initial findings suggest that the concept has significant added-value for the target group of students with professional experience, provided that the practioner comes from the same field as the students.
\end{abstract}

\section{Keywords}

external practitioners, cooperative teaching, part-time studies, innovative teaching approach

\section{Einführung}

Dieser Beitrag stellt erste Erfahrungen mit einer neuen kooperativen Lehrform unter der Bezeichnung „Tandem Teaching“ vor. Die praktische Erprobung erfolgt im berufsbegleitenden Studiengang „Soziale Arbeit mit dem Schwerpunkt Jugendarbeit" an der Hochschule Kempten. Dieser ist für berufserfahrene Fachkräfte aus dem Handlungsfeld der Jugendarbeit konzipiert, die ihre bereits erworbenen Kompetenzen (u. a. aus vorangegangenen Ausbildungen) für dieses Studium geltend machen können. Ziel dieses Werkstattberichts ist, das innovative Potential des Tandem Teaching für die wissenschaftliche Weiterbildung, vor allem im Hinblick auf die Theorie-Praxis-Verzahnung, die Professionalitätsentwicklung und die Ausrichtung der Hochschuldidaktik insbesondere auf diese neue Zielgruppe lebenslangen Lernens, zu verdeutlichen. Dies geschieht gestützt auf erste Befunde aus der Evaluation. 


\section{Theoretischer Hintergrund}

Das hier vorgestellte Konzept vereint zwei Ideen, die der kooperativen Lehre und die der Einbindung hochschulexterner Praktiker/innen in die Lehre. Beide Ideen für sich sind nicht neu, doch wurde über die Kombination dieser beiden Lehrarrangements bisher nicht publiziert.

Unter Kooperativer Lehre wird nach WENZLAFF et al. (2002) ein Lehrarrangement verstanden, bei dem zwei oder mehrere Lehrpersonen in eine kollaborative Beziehung treten, um gemeinsam zu arbeiten und zu lehren, wobei ein Ergebnis entsteht, welches eine Lehrperson allein nicht hätte erreichen können (vgl. ebd., S. 14). Wichtig ist dabei die gleichzeitige Anwesenheit und Face-to-faceBegegnung im realen Raum (COOK \& FRIEND, 1995). Die Wirksamkeit und der Nutzen kooperativ gestalteter Lehrarrangements sind empirisch gut erforscht und bestätigt. Dabei fokussieren einige Autorinnen/Autoren die Lernerfolge der Studierenden, betonen aber ganz unterschiedliche Effekte, wie zum Beispiel die TheoriePraxis-Verzahnung oder Interdisziplinarität (BUCKLEY, 2000; JESSENMARSHALL \& LESCINSKY, 2011), die Abwechslung und damit die Vermeidung von Langeweile (BERGMAN, 1990) und die gesteigerte didaktische Qualität der Lehre (PLANK, 2013). Auch fördert ein möglicher Kontrast der Standpunkte zweier Lehrpersonen die Fähigkeiten in Analyse sowie Synthese und schult das selbständige und kritische Denken (vgl. BUCKLEY, 2000, S. 13 f.). Andere Ansätze heben die integrative Funktion kooperativer Lehrformen hervor (HALFHIDE, 2009). Auch die Lehrenden werden explizit in den Blick genommen. Zum Beispiel kann diese Art der Lehre die Begeisterungsfähigkeit der Lehrenden für ihre Profession beleben (BACHARACH \& HECK, 2007).

Die zweite grundlegende Idee ist die Einbindung hochschulexterner Praktiker/innen in die Lehre. Das bedeutet, dass eine Lehrperson ihren Arbeitsschwerpunkt in der beruflichen Praxis hat und konsequent ihre berufliche Perspektive in die Lehre einbringt. Praktiker/innen als Lehrende unterstützen die Studierenden dabei, auch ihre eigenen in der Praxis gewonnenen Erfahrungen mit dem an der Hochschule erworbenen Wissen zu verknüpfen (vgl. NIGGLI, HEIMGARTNER- 
MORONI, GUT \& BERTSCHY, 2012, S. 274) und erleichtern es ihnen, Theorie und Praxis nicht als gegensätzlich anzusehen, sondern entsprechend DEWE, FERCHHOFF, SCHERR \& STÜWE (2001) als unterschiedliche Verhältnisse von Entscheidungs- und Begründungswissen. Das Potential dieser Art von Lehre liegt auch darin, dass es in jedem Bereich der beruflichen Praxis Ideen und Prinzipien gibt, welche nie Gegenstand wissenschaftlicher Betrachtung waren und grundsätzlich nicht wissenschaftlich begründbar sind. Nichtsdestoweniger stellen diese Ideen und Prinzipien das angesammelte Erfahrungswissen dar und sind ebenso wichtig für die Praxis wie die Theorie oder empirische Prinzipien (vgl. SHULMAN, 1986, S. 11). Ein weiterer wichtiger Vorteil der Einbindung von Praktikerinnen/Praktikern in die Lehre für die Zielgruppe der berufserfahrenen Studierenden mit ihren höchst heterogenen Berufs- und Bildungsbiografien ist, dass diese aufgrund ihrer eigenen Erfahrungen ,die Lern-Ausgangslage und den Praxiszugang zu einem Thema von Lernenden besser einschätzen und auch würdigen [können]“ (CENDON \& FLACKE, 2013, S. 37).

Die kooperative Lehre und die Einbindung hochschulexterner Praktiker/innen werden nun zu einem Lehrkonzept mit der Bezeichnung „Tandem Teaching“ verwoben. Es wird definiert als kooperatives Lehrformat, bei dem genau zwei Lehrpersonen aus dem konkreten berufspraktischen Bereich und aus der theoretischwissenschaftlichen Perspektive eines Handlungsfeldes in einem nicht hierarchischen Verhältnis zwischen Theorie und Praxis gemeinsam anwesend lehren und auch Konzeption, Vorbereitung und Ausgestaltung der Lehrveranstaltung kollegial übernehmen. In Abgrenzung zu anderen kooperativen Lehrformaten wie zum Beispiel Co- oder Teamteaching wird beim Tandem Teaching der Akzent auf die Bedeutung der gemeinsamen gleichzeitigen Anwesenheit gelegt. Denn nur so sind das Praxis- als Entscheidungswissen und das Theorie- als Begründungswissen stets in der Lehre repräsentiert. Entsprechend der unterschiedlichen Verhältnisse zwischen Entscheidungs- und Begründungswissen ist es selbstverständlich, dass auch Praktiker/innen auf theoretische Bezugspunkte zurückgreifen, entsprechend ist auch die Trennung zwischen Theorie und Praxis bei den Lehrenden nicht trennscharf. Wesentlich mit diesem Lehrformat verbundene Bedingung und Herausforderung ist, 
dass die Lehrkonstellation nicht hierarchisch verstanden wird, das heißt, dass beide Tandempartner/innen gleichrangig unterrichten, führen, assistieren und fördern (vgl. KRICKE \& REICH, 2016, S. 43). Dementsprechend darf auch das Verhältnis zwischen Theorie und Praxis kein hierarchisches sein, sondern die „Gleichwertigkeit von Theorie und Praxis [...] gelebtes Prinzip“ (CENDON \& FLACKE, 2013, S. 37). Nur wenn die Hierarchielosigkeit als Prämisse gesetzt wird, kann zum Beispiel die überzeugende Würdigung der Praxiserfahrungen der Studierenden erfolgen oder echte Kooperation innerhalb des Tandems vorgelebt werden. Basis dafür ist eine funktionierende Beziehungsebene, welche sich durch gegenseitige Anerkennung und Wertschätzung der Personen und ihrer jeweiligen Expertise auszeichnet (vgl. ebd., S. 38). Dies erfordert von den Beteiligten neben ,intensiver Kommunikationsarbeit" (ebd.) auch Aufgeschlossenheit, Phantasie und Kreativität sowie die Bereitschaft die eigene Didaktik zu hinterfragen (BUCKLEY, 2000). Insgesamt ist diese anspruchsvolle Form der Kooperation (vgl. HALFHIDE, 2009, S. 109) überaus arbeitsintensiv (vgl. PARSON, 1994, S. 63) und bedarf einer sehr sorgfältigen Planung, wenn gelingen soll, dass sie eben mehr ist als die bloße Anwesenheit zweier Lehrpersonen (vgl. LAUGHLIN, NELSON \& DONALDSON, 2011, S. 12).

\section{Umsetzung}

Im Folgenden wird der Prozess der Umsetzung des Tandem Teaching beschrieben. Dabei wird das Vorgehen anhand der aus den theoretischen Hintergründen abgeleiteten Annahmen reflektiert. Dies geschieht in beschreibender Form auf Grundlage der Dokumentation der gemeinsamen Vorbereitung der Lehrenden auf die Lehrveranstaltung. Diese wurde von den Autoren dieses Beitrags, welche den Studiengang im Rahmen des Forschungsprojekts „Jugendarbeit mit Perspektive (JumP)“ wissenschaftlich begleiten, durchgeführt. Des Weiteren erfolgt die Analyse von Evaluationsdaten, die nach Durchführung der Lehrveranstaltung im Sommer 2019 durch eine quantitative Studierendenbefragung mit einem eigens dafür konstruierten Fragebogen erhoben wurden. Aus den theoretischen Vorüberlegungen zur ko- 
operativen Lehre und der Einbindung einer Lehrperson aus dem beruflichen Praxisfeld in das Lehrtandem lassen sich drei Annahmen ableiten, welche die speziellen Ansprüche und Anforderungen berufserfahrener Studierender an eine Didaktik der wissenschaftlichen Weiterbildung reflektieren.

1. Tandem Teaching erreicht die direkte Verknüpfung von wissenschaftlichtheoretischen und handlungspraktischen Perspektiven und trägt so dazu bei, neues Wissen und erweiterte Möglichkeiten für praktisches Handeln $\mathrm{zu}$ generieren.

2. Eine Hierarchisierung zwischen Theorie und Praxis wird durch die gezielte Vorbereitung des Tandems vermieden.

3. Das Tandem Teaching unterstützt in besonderem Maße diejenigen Studierenden bei der Aneignung theoretischer Wissensbestände, die dem akademischen Lernen eher distanziert gegenüberstehen.

Vor diesem Hintergrund begann zunächst die Findungsphase des Tandems. Die Gewinnung der hochschulischen Lehrperson gestaltete sich unproblematisch, da eine interessierte Lehrperson bereits in den berufsbegleitenden Studiengang „Soziale Arbeit mit dem Schwerpunkt Jugendarbeit“ eingebunden war. In Vorgesprächen zur Umsetzung des Tandem Teaching wurde deutlich, dass die Auswahl der Tandempartnerin oder des Tandempartners der Lehrperson überlassen werden sollte, um eine fachlich gemeinsame Perspektive im beruflichen Handlungsfeld, einen persönlichen Bezug zu den Inhalten der Lehrveranstaltung sowie eine auf Sympathie basierende Beziehungsebene im Tandem sicherzustellen und so die Grundvoraussetzung für eine gelingende Zusammenarbeit zu gewährleisten. Die Akquirierungsversuche einer Lehrperson aus der Praxis stießen auf geringe Resonanz, obwohl die Vergütung überdurchschnittlich hoch angesetzt wurde. Nach längerer Suche konnte eine qualifizierte Person aus dem beruflichen Praxisfeld der Jugendarbeit gewonnen werden.

Um die theoretischen Überlegungen aufzunehmen, wurde ein Vorbereitungstreffen durchgeführt, in welchem die Erwartungen und Befürchtungen bezüglich der Lehrveranstaltung thematisiert wurden. Die Lehrperson aus dem Handlungsfeld formu- 
lierte vor allem die Sorge, dass sie die an sie gerichteten Erwartungen möglicherweise nicht erfüllen kann. Die Lehrperson aus der Hochschule legte den Fokus bei der Formulierung von Befürchtungen eher auf die Organisation eines reibungslosen Ablaufs. Dies veranlasste sie auch dazu die Moderation zu übernehmen, um die komplexe Kommunikationsstruktur des berufsbegleiten Studienformates darzustellen, und benannte aus ihrer Sicht diesbezügliche konkrete Arbeitsaufträge. Allen Beteiligten wurde ersichtlich, dass die vorhandene Kommunikationsstruktur bereits die Entstehung einer hierarchischen Zuordnung zwischen Hochschul- und Praxispart des Tandems begünstigt. Wahrnehmbar war, dass die Lehrperson aus dem beruflichen Arbeitsfeld zum ersten Mal mit der Komplexität des berufsbegleitenden Studienformates in Berührung kam, da sie sich überrascht über den Mehraufwand bzw. die Vielschichtigkeit ihrer Rolle (z. B. Verteilung und Betreuung von Studienarbeiten und Seminarprotokollen, Begleitung des selbstorganisierten Lernens, usw.) äußerte.

Nach der gemeinsamen Vorbereitung erfolgte die Durchführung des Tandem Teaching. Den Schwerpunkt bildete dabei eine dreitägige PräsenzBlockveranstaltung mit 22 Teilnehmenden des zweiten Semesters des berufsbegleitenden Bachelor-Studiengangs ,Soziale Arbeit mit dem Schwerpunkt Jugendarbeit" im Sommersemester 2019. In der didaktischen Umsetzung wurden traditionelle Lehrmethoden wie beispielsweise der klassische Vortrag mit dem Ziel aufgebrochen, die Erfahrungen aus dem Feld der Studierenden miteinzubeziehen. In der vorab angelegten Aufgabenstellung, die Leitlinien ihrer eigenen pädagogischen Einrichtung im geschichtlichen Kontext zu erforschen, konnten persönliche Erkenntnisse der Studierenden direkt in die Veranstaltung eingebettet und historisch sowie theoretisch begründet werden. Es folgten Einzel- und Gruppenarbeiten sowie Kurzimpulse der Lehrenden, die in Formaten wie im Fishbowl oder einer Podiumsdiskussion dialogisch bearbeitet wurden.

Im direkten Anschluss an die letzte Einheit des Tandem Teaching wurde die Lehrveranstaltung per Fragebogen evaluiert. Im Folgenden werden Ergebnisse dargestellt, die mit den in diesem Beitrag formulierten Annahmen in Zusammenhang stehen. So gaben auf die Frage, ob die Gestaltung der Lehre durch zwei Lehrende, 
die gleichzeitig anwesend sind, erkennbare Vorteile bringt (Antwortmöglichkeiten von 1 ,trifft gar nicht zu“ bis 6 ,trifft voll zu“, $M W=5.18, S D=1.29$ ) 63.6 Prozent der Befragten an, dass dies voll zutreffe. Zwischen dieser Beurteilung und dem subjektiv eingeschätzten Lernerfolg besteht mit r=.59 ein starker Zusammenhang. ${ }^{3}$

Bei der sechsstufig skalierten Frage, ob sie bei der Lehrveranstaltung das Gefühl hatten, dass Gleichrangigkeit zwischen Theorie und Praxis herrschte ${ }^{4}$, lagen die Antworten von 40.9 Prozent der Studierenden in der obersten Kategorie (,trifft voll $\left.\mathrm{zu}^{6}\right)$. Der Mittelwert MW=4.91 ( $\left.\mathrm{SD}=1.29\right)$ für dieses Item liegt deutlich über dem Skalenmittel von MW=3.50.

Auf die Frage, inwiefern sie Berührungsängste haben, wenn es um die Aneignung theoretischen Wissens geht (Antwortmöglichkeiten von 1 , trifft gar nicht zu“ bis 6 „trifft voll $\left.\mathrm{zu}^{\prime \prime}\right)$, lagen 54.5 Prozent aller Studierenden mit ihrer Antwort in der untersten Kategorie, gaben also an, dass dies für sie gar nicht zutreffe (MW=1.82, $\mathrm{SD}=1.26, \mathrm{~N}=22$ ).

Betrachtet man diese Frage differenziert nach Berufsbiographie der Studierenden, liegt der Mittelwert bei denjenigen, die dem Arbeitsfeld Jugendarbeit und dem akademischen Lernen eher ferner stehen (heterogene Bildungsbiographie) - zur Kategorisierung wird hier die Anzahl an Berufsjahren außerhalb des Arbeitsfelds des Studiengangs zwischen Erwerb der Hochschulzugangsberechtigung und Aufnahme des Studiums herangezogen -, mit MW=2.63 ( $\mathrm{SD}=1.51, \mathrm{~N}=8$ ) um mehr als einen Skalenpunkt höher als bei den Studierenden mit homogener Berufsbiographie - also die keine Berufserfahrung außerhalb des Handlungsfeldes besitzen $(\mathrm{MW}=1.36, \mathrm{SD}=.84, \mathrm{~N}=14)$. Letztere sind auch diejenigen Studierenden, die ihre beruflichen Erfahrungen nicht im selben Handlungsfeld wie der Praxispart des

${ }^{3}$ Korrelation nach Pearson, $\mathrm{p}=.01$; die Skala „Lernerfolg“ beinhaltet neun Items, Beispielitem: „Ich habe den Eindruck, in dieser Veranstaltung viel gelernt zu haben“, Antwortmöglichkeiten von 1 ,trifft gar nicht zu“ bis 6 ,trifft voll zu“, $\mathrm{MW}=5.13, \mathrm{SD}=.75, \mathrm{~N}=22$.

${ }^{4}$ Itemtext: „Bei der Lehrveranstaltung hatte ich das Gefühl, dass eine Gleichrangigkeit zwischen Theorie und Praxis herrschte." 
Tandems gemacht haben. Dieser Unterschied kann gegen den Zufall abgesichert werden $\left(\mathrm{F}_{(1,21)}=6.52, \mathrm{p}=.02\right)$.

Auch wurden die Studierenden um ihre Einschätzung der Gesamtbilanz der Lehrveranstaltung gebeten (Skala: 3 Items, Beispielitem: „Die Lehre an der Hochschule sollte öfter durch ein Tandem gestaltet werden", Antwortmöglichkeiten von 1 „trifft gar nicht zu“ bis 6 ,trifft voll zu“, MW=5.09, SD=1.14, N=22). Der Mittelwertunterschied zwischen Studierenden mit homogener ( $M W=4.46, S D=1.19$, $\mathrm{N}=8)$ und heterogener ( $\mathrm{MW}=5.46, \mathrm{SD}=.97, \mathrm{~N}=14)$ Berufsbiographie beträgt einen Skalenpunkt und ist signifikant $\left(\mathrm{F}_{(1,21)}=4.64, \mathrm{p}=.01\right)$.

\section{Fazit}

Die Befragung der Studierenden kann die oben beschriebene erste Annahme zur Generierung neuen Wissens vorerst nicht empirisch bestätigen. Dies liegt an den mit diesem Werkstattbericht verbundenen Limitationen, dass sich die hier gemachten Aussagen lediglich auf die subjektiven Einschätzungen der Studierenden einer einzigen Lehrveranstaltung stützen und die Fallzahl $(\mathrm{N}=22)$ relativ klein ist. Jedoch zeigen die ersten Ergebnisse, dass die große Mehrheit das Tandem Teaching sehr positiv beurteilt. Auch gibt es Hinweise darauf, dass die Studierenden, die diesem Lehrkonzept positiv gegenüberstehen, auch ihren Lernerfolg erheblich höher einschätzen.

Bezogen auf die zweite Annahme zur Vermeidung von hierarchischen Zuordnungen im Tandem zeigte sich, dass obwohl die Lehrperson der Hochschule ein emanzipatorisches Verständnis von Zusammenarbeit und eine Lehre „auf Augenhöhe“ vertritt und von Beginn an eine funktionierende Beziehungsebene vorhanden war, eine Hierarchisierungstendenz zwischen Theorie- und Praxispart zu beobachten war. Die Lehrperson aus dem beruflichen Handlungsfeld ist mit hochschulinternem Wissen und den Organisationsstrukturen nicht vertraut, muss sich in ein bereits bestehendes System hineinfinden und begibt sich allein dadurch in ein Abhängigkeitsverhältnis. Dies wirkte sich jedoch aus Sicht der Studierenden nicht negativ 
auf die angestrebte Gleichrangigkeit zwischen der wissenschaftlich-systematischen Theorieperspektive und der Perspektive des beruflichen Handlungsfeldes während der Lehrveranstaltung selbst aus.

Was die dritte Annahme zur besonderen Eignung des Tandem Teaching für berufserfahrene Studierende betrifft, zeigen die ersten Ergebnisse aus der Evaluation, dass sich die erwarteten Berührungsängste der berufserfahrenen Studierenden mit der Aneignung systematisch-theoretischen Wissens empirisch bestätigen lassen. Insgesamt schätzen die Studierenden ihre Berührungsängste mit Theorie zwar gering ein, allerdings sind diese bei den Studierenden mit heterogener Berufsbiographie wesentlich größer. Hinsichtlich der Gesamtbilanz der Studierenden zum Tandem Teaching ergibt sich ein negativer Zusammenhang zwischen der Zugehörigkeit zur Gruppe der Studierenden mit heterogener Berufsbiographie und den übrigen Studierenden. Die dargestellten empirischen Ergebnisse in Bezug auf die Studierenden zeigen, dass die positiven Effekte des Tandem Teaching nur spürbar zur Geltung kommen, wenn berufsbegleitend Studierende auf Praktiker/innen treffen, die berufliche Erfahrungen im selben Arbeitsfeld haben. Somit kann das Format vor allem berufsbegleitenden Studiengängen empfohlen werden, die sich auf klare Berufsfelder beziehen und stark spezialisiert sind.

Beim Prozess zur Gewinnung der Tandempartner/innen zeigte sich, dass trotz angemessener Vergütung wenig Interesse bestand, am Projekt mitzuwirken. Möglicherweise müssen die Lehrpersonen aus dem beruflichen Handlungsfeld über hohe fachliche Kompetenzen verfügen und sich ihrer Wissensbestände sehr sicher sein wie die im vorliegenden Projekt involvierte Person - um nicht zu befürchten, sich der Lehrperson der Hochschule fachlich unterordnen zu müssen. Es ist davon auszugehen, dass deshalb eher Personen dazu bereit sind, sich zu engagieren, die sich bereits intensiver mit systematisch-theoretischen Bezügen auseinandergesetzt haben und eventuell bereits Erfahrung in der (wissenschaftlichen) Weiterbildung sammeln konnten. 


\section{Literaturverzeichnis}

Bacharach, N. \& Heck, T. W. (2007). Co-Teaching In Higher Education. Journal of College Teaching \& Learning, 4(10), 19-26.

Bergman, F. L. (1990). Restructuring the Curriculum. Tandem Teaching Relieves Boredom, Maximizes Class Time. NASSP Bulletin, 74(527), 89-94.

Buckley, F. J. (2000). Team teaching. What, why, and how? Thousand Oaks, Ca: Sage Pub.

Cendon, E. \& Flacke, L. B. (2013). Praktikerinnen und Praktiker als hochschulexterne Lehrende in der wissenschaftlichen Weiterbildung. Eine notwendige Erweiterung des Lehrkörpers. Hochschule und Weiterbildung, 13(1), 36-40.

Cook, L. \& Friend, M. (1995). Co-Teaching: Guidelines for Creating Effective Practices. Focus on Exceptional Children, 28(3), 1-16.

Dewe, B., Ferchhoff, W., Scherr, A. \& Stüwe, G. (2001). Professionelles soziales Handeln. Soziale Arbeit im Spannungsfeld zwischen Theorie und Praxis. Weinheim: Juventa.

Halfhide, T. (2009). Teamteaching. In S. Fürstenau \& M. Gomolla (Hrsg.), Migration und schulischer Wandel: Unterricht (S. 103-120). Wiesbaden: Verlag für Sozialwissenschaften.

Jessen-Marshall, A. \& Lescinsky, H. L. (2011). Origins. Team Teaching in the Sciences. In K. M. Plank (Hrsg.), Team teaching. Across the disciplines, across the academy (S. 13-35). Sterling, Va: Stylus Pub.

Kricke, M. \& Reich, K. (2016). Teamteaching. Eine neue Kultur des Lehrens und Lernens. Weinheim: Beltz.

Laughlin, K., Nelson, P. \& Donaldson, S. (2011). Successfully Applying Team Teaching with Adult Learners. Journal of Adult Education, 40(1), 11-18. 
Niggli, A., Heimgartner-Moroni, S., Gut, R. \& Bertschy, B. (2012). Navigieren zwischen Theorie und Praxis in Mentoratsgesprächen mit Lehramtstudierenden. In C. Nerowski, T. Hascher, M. Lunkenbein \& D. Sauer (Hrsg.), Professionalität im Umgang mit Spannungsfeldern der Pädagogik (S. 273-284). Bad Heilbrunn: Verlag Julius Klinkhardt.

Parson, S. R. (1994). Program Development, Team Instruction, and the Impact on Faculty Members. NASSP Bulletin, 78(559), 62-64.

Plank, K. M. (2013). Team Teaching. Idea Paper 55, IDEA Center Inc., Otterbein University. http://files.eric.ed.gov/fulltext/ED565291.pdf, Stand vom 26. Juni 2019.

Reich, K. (2016). Team Teaching. In: Methodenpool Universität Köln. http://methodenpool.uni-koeln.de/download/teamteaching.pdf, Stand vom 26. Juni 2019.

Shulman, Lee S. (1986). Those Who Understand: Knowledge Growth in Teaching. Educational Researcher, 15(2), 4-14.

Wenzlaff, T. L., Berak, L., Wieseman, K. C., Monroe-Baillargeon, A., Bacharach, N. \& Bradfield-Kreider, P. (2002). Walking our talk as educators: Teaming as a best practice. In E. Guyton \& J. D. Rainer (Hrsg.), Research on meeting and using standards in the preparation of teachers (S. 11-24). Dubuque, la: Kendall/Hunt. 


\section{Autoren}

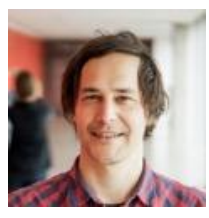

Dipl.-Soz. Andreas ROTTACH || Hochschule Kempten \|

Bahnhofstraße 61, D-87435 Kempten (im Allgäu)

www.hs-kempten.de

andreas.rottach@hs-kempten.de

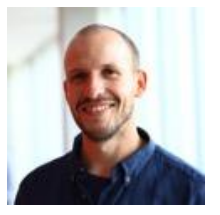

Micha JUNG (M.A.) \| Hochschule Kempten \| Bahnhofstraße 61, D-87435 Kempten (im Allgäu)

www.hs-kempten.de

micha.jung@hs-kempten.de

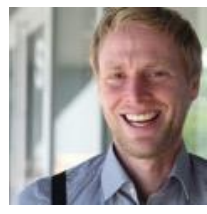

Thomas MILLER (M.A.) || Hochschule Kempten \|

Bahnhofstraße 61, D-87435 Kempten (im Allgäu)

www.hs-kempten.de

thomas.miller@hs-kempten.de 